https://doi.org/10.7559/gestaoedesenvolvimento.2019.374

Data de receção: 13/03/2019 Data de aceitação: 24/06/2019

\title{
EMPOWERMENT ESTRUCTURAL: POTENCIANDO LA CAPACIDAD INNOVADORA DE LAS ORGANIZACIONES
}

\section{STRUCTURAL EMPOWERMENT: ENHANCING THE INNOVATIVE CAPACITY OF ORGANIZATIONS}

\author{
José Román ${ }^{1}$ orcid.org/0000-0003-3751-1536 \\ Paulo Almeida Pereira ${ }^{2}$ orcid.org/0000-0002-3941-8274 \\ Célia Ribeiro ${ }^{3}$ orcid.org/0000-0002-1000-6890
}

\begin{abstract}
Resumo: O objetivo fundamental deste trabalho de investigação é identificar o nível de empowerment estrutural em colaboradores pertencentes a empresas com características inovadoras.

Desenhou-se um estudo de caráter exploratório, descritivo e correlacional com a utilização de métodos de investigação quantitativa. A amostra é constituída por 90 colaboradores pertencentes a três empresas consideradas como possuindo características inovadoras, do distrito de Viseu, Portugal.

Os resultados permitem verificar o impacto do empowerment estrutural nas características sociodemográficas e profissionais dos colaboradores nas três empresas consideradas como inovadoras, em função de quatro dimensões: oportunidade, informação, apoio $e$ recursos. Efetivamente, estas quatro dimensões apresentaram relações significativas entre si e contribuem positivamente para a perceção dos colaboradores sobre o empowerment estrutural.
\end{abstract}

\footnotetext{
${ }^{1}$ Mestre em Gestão, Especialização em Gestão de Negócios. Professor Universitário da Área de Gestão da FACEN - Universidade Nacional de Assunção, Gestor da Área de Estratégia e Marketing. E-mail: roman.jose07@gmail.com

${ }^{2}$ Professor Auxiliar da Universidade Católica Portuguesa - Viseu - Instituto de Gestão e das Organizações da Saúde. E-mail: ppereira@ viseu.ucp.pt

3 Doutorada em Psicologia, área de especialidade em Psicologia Pedagógica. Professora Auxiliar da Universidade Católica Portuguesa - Viseu. E-mail: cribeiro@viseu.ucp.pt
} 


\section{Jose Román, Paulo Almeida Pereira e Célia Ribeiro}

Verificou-se também que os colaboradores que têm menor experiência profissional e menos tempo de serviço nas empresas apresentam um maior nível de empowerment estrutural.

Palavras-chave: Empowerment estrutural, inovação, motivação, autonomia.

Abstract: The main goal of this work is to identify the level of structural empowerment of employees who work for companies with innovative characteristics. An exploratory, descriptive and correlational with the use of quantitative research methods study was designed. The sample is composed by 90 employees of companies that were considered as presenting innovative characteristics, in the Viseu district, in Portugal. Results demonstrate the impact of structural empowerment in the sociodemographic and professional characteristics of employees, according to four dimensions: opportunity, information, support and resources. These four dimensions actually show significant relationships with each other and contribute positively to the employees' perception of structural empowerment.Moreover, it was verified that employees who have lower professional experience and a shorter period of employment in companies present a higher level of structural empowerment.

Keywords: Structural empowerment, innovation, motivation, autonomy.

\section{INTRODUCCIÓN}

Ante la complejidad de la creciente competencia sumada al incremento de la demanda y las exigencias del mercado en cuanto a calidad, rapidez, flexibilidad, funcionalidad y costos competitivos han puesto a las organizaciones, independientemente de su tamaño, en un proceso de cambio acelerado tanto en sus procesos organizacionales y en las personas involucradas en las mismas.

En este contexto, las tendencias en el campo de la información y avances en telecomunicaciones así como en la llamadas nuevas filosofías, métodos, técnicas que apuntan hacia un futuro en el que las habilidades, destrezas, competencias y actitudes de las personas deben responder de 
manera innovadora, rápida y eficiente ante los cambios que emergen, ya que la supervivencia de las mismas dependerá de los cambios en el entorno global.

Las empresas u organizaciones requieren una nueva forma de gestión para adaptarse a los cambios y sobrevivir. Una de estas es el empowerment, una nueva forma de orientar el trabajo de las organizaciones hacia un futuro próspero e innovador.

Así, en este nuevo contexto que emerge planteamos la siguiente cuestión de investigación: Las organizaciones innovadoras potencian el empowerment estructural en sus colaboradores?

\section{REVISIÓN DE LA LITERATURA}

\subsection{Una visión emergente}

Un ambiente competitivo, dinámico y complejo, donde la necesidad de innovación es un factor determinante para el éxito de las organizaciones, exige que las personas estén cada vez más involucradas en procesos de decisión asociados a las estrategias organizacionales (Ribeiro \& Almada, 2001). Es ahí donde nace el empowerment, con el objetivo de capacitar a las personas para esos procesos decisorios en la organización, buscando como resultado las transformación de las empresas para adaptarlas a ambientes competitivos, complejos y dinámicos (Ribeiro \& Almada, 2001).

A lo largo de los años, diferentes abordajes han sido particularmente influyentes en diferentes momentos (Figura 1). 


\section{Figura I}

Los diferentes enfoques del trabajo: cada una implica un equilibrio diferente entre control y compromiso (Slack, Chambers, Harland, Harrison \& Johnston, 1997,

p.312).

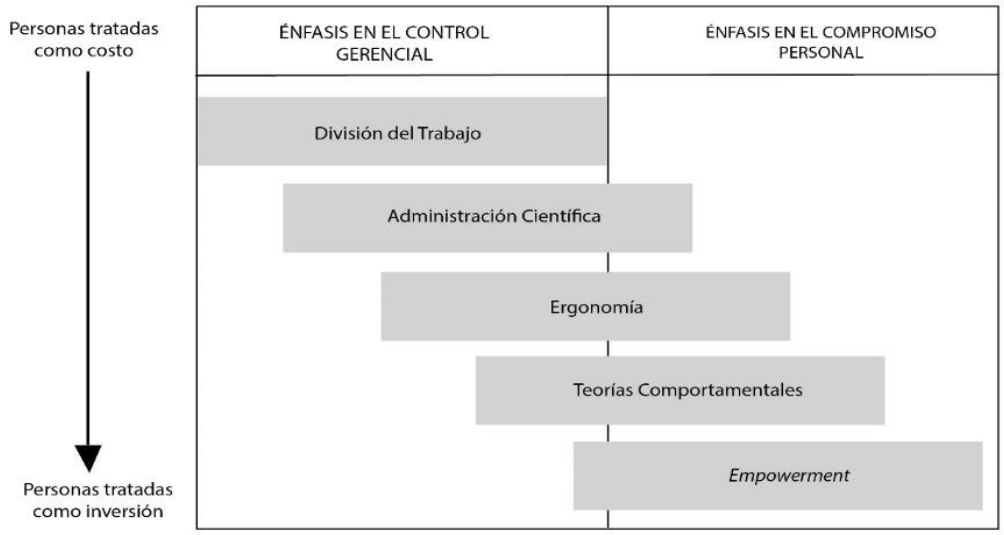

\subsection{El concepto de Empowerment}

La dura competencia en la actualidad, la globalización de los mercados y las economías que fluctúan impredeciblemente, han llevado a las empresas en todo el mundo a buscar soluciones empresariales basados es nuevas y modernas teorías que mejoren su desempeño de manera global. Los presidentes de las organizaciones, según Salazar y Molano (2000), definen esta situación como un torneo de grandes ligas, de grandes competencias mundiales, el cual plantea cada día para las organizaciones, nuevos desafíos para los administradores.

En esta óptica, el empowerment surge como una solución a los viejos problemas del taylorismo y de la burocracia en los locales de trabajo, donde la creatividad era reprimida y los trabajadores se convertían en objetos de venta (Wilkinson, 1998).

Es así que, las modernas organizaciones están dejando atrás las estructuras tradicionales organizadas en órganos y puestos y prefieren utilizar estructuras integradas por equipos, hoy en día las organizaciones están juntando e integrando a su personal en lugar de separarlos e individualizar el trabajo. 
La delegación de funciones a las personas de todas las áreas, así como la implantación de una cultura participativa es la base de las organizaciones que buscan descentralizar el poder e información entre sus miembros; el mismo Chiavenato (2009) enuncia el empowerment como el facultamiento en la toma de decisiones o delegación de autoridad.

Así, el empowerment significa dar poder a los empleados para aprovechar al máximo el talento colectivo, el secreto consiste en utilizar todo el personal, todas las habilidades, todo el tiempo, dar autoridad y recursos a las personas y dejarlas actuar (Chiavenato, 2009).

De acuerdo con Spreitzer (2008), actualmente, más del 70\% de las organizaciones adoptan algún tipo de iniciativa de empowerment en por lo menos una parte de su fuerza de trabajo. Para alcanzar el éxito en el contexto competitivo actual, las organizaciones necesitan del conocimiento, de las ideas, de la creatividad y de la energía de cada colaborador.

En este sentido, se puede afirmar que, el papel de la alta gerencia es crear las condiciones necesarias para la eficacia del trabajo, asegurando que los colaboradores tengan acceso a información, apoyo y a recursos necesarios para la ejecución de sus tareas y, a las oportunidades para el desarrollo y crecimiento personal y profesional. Como consecuencia de elevados niveles de empowerment, los colaboradores experimentarán sentimientos positivos en su trabajo y serán más eficaces para alcanzar las metas organizacionales (Gouveia, 2013).

Según Wilson (1996) el empowerment presenta la siguiente dinámica: 
Figura II

Círculo del empowerment (Wilson, 1996, p. 24).

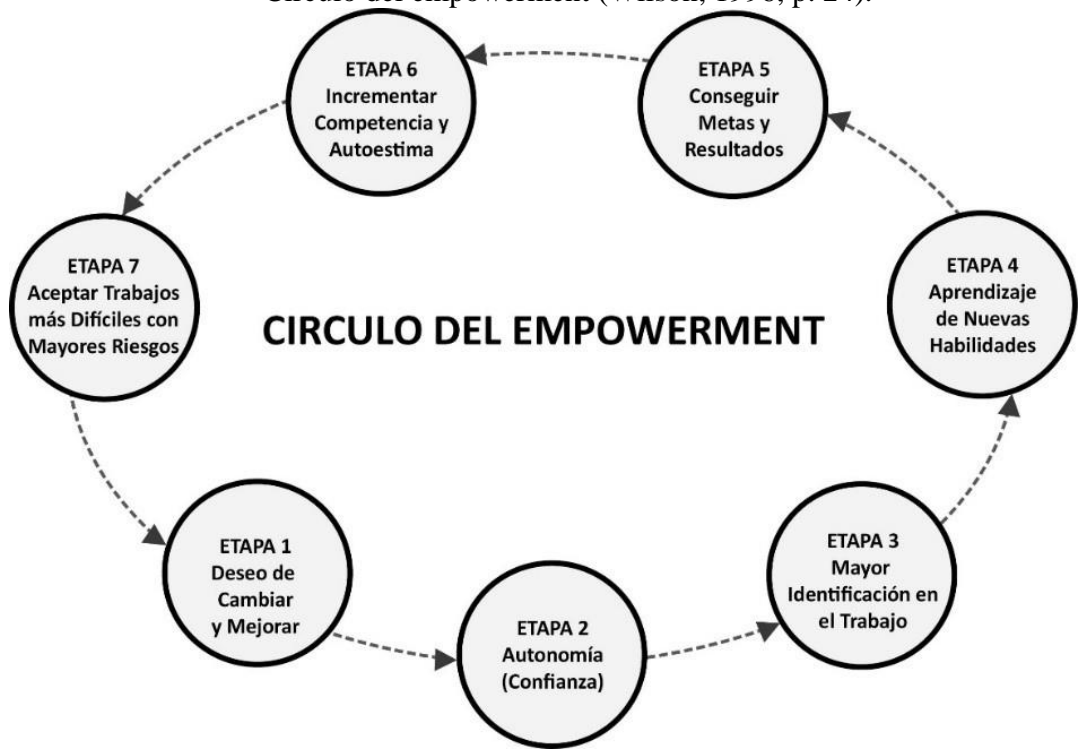

La atracción que supone el empowerment para los individuos y empresas, enmascara muchos de los factores que ayudan o impiden su introducción efectiva en las empresas. Se da por sentado que los beneficios del empowerment son tan importantes que las personas se convierten de inmediato en discípulos. Sin embargo, cualquier empresa que considera seriamente la idea de introducir esta herramienta debe pensar y evaluar algunos factores que ayudan a su efectiva introducción (Wilson, 1996).

\subsection{Rol estratégico de la innovación}

Cuando se habla de innovación se debe entender que, detrás de todo proceso innovador están involucradas las personas. La innovación nace de ideas y propuestas concebidas y expresadas por personas, se concreta en planes o proyectos elaborados por personas y son personas las que llevan los proyectos innovadores a la práctica. En todos los casos, serán personas quienes de forma individual o colectiva, abordarán problemas o tomarán decisiones que favorecerán o frenarán la innovación. Desde el punto de 
vista empresarial, es por tanto muy importante tener en cuenta este papel central de la persona para que sus procesos de innovación funcionen eficazmente (Gisbert \& Velasco, 2007).

Como se menciona en el Manual de Oslo (2006), una gran parte del conocimiento propio sobre la innovación se encuentra depositado en las personas y su experiencia, en este sentido, el capital humano desempeña un papel fundamental tanto a nivel organizacional como a nivel global. La teoría de los entornos innovadores propone que la innovación es resultado de procesos de aprendizaje colectivo en contextos sociales, culturales e institucionales específicos en los cuales las empresas con sus contactos y relaciones-redes, acceden o propician las innovaciones (Gisbert \& Velasco, 2007).

Por eso, deben tenerse condiciones o ambientes adecuados en las organizaciones para que las innovaciones se gesten y desarrollen. Como aprendizaje colectivo, la innovación no es un proceso lineal, es una espiral, un proceso mental que revisa los asuntos una y otra vez aunque desde diferentes perspectivas, cada vez más cerca del objetivo (Arborniés, 2009).

De este modo, una organización creativa buscará promover globalmente una cultura y un clima empresarial que potencie la generación de ideas, planteará la actividad innovadora desde una perspectiva estratégica que permita aprovechar las ideas generadas y procurará establecer mecanismos estructurales adecuados para el soporte y la implantación de la estrategia de innovación (Gisbert \& Velasco, 2007).

Las empresas innovadoras son más flexibles, se adaptan más rápido a los cambios del entorno y responden de una manera más rápida y mejor a las necesidades cambiantes de la sociedad en su conjunto para obtener mejores resultados (Drucker, 1985; Miles \& Snow, 1978).

Debido a estas situaciones mencionadas anteriormente, la innovación hoy en día es considerada como un factor clave para la supervivencia y éxito de las empresas (Damanpour \& Gopalakrishnan, 2001; Rogers, 1983). Tal como lo afirmaba Schumpeter (1935), las empresas son innovadoras o no existen.

Como se observa en la figura 4, es importante destacar que algunos factores fundamentales para la generación de una cultura basada en la 
innovación son: el comportamiento, la estructura, la estrategia, los mecanismos de apoyo y la comunicación. Estos factores involucran dentro de sí las distintas maneras de desempeño de las empresas, incluso explican las estrategias y operaciones diarias de las empresas dentro de cada uno de estos factores (Martins \& Terblanche, 2003).

\section{Figura III}

Determinantes que influencian a la innovación. Elaboración propia basada en Martins y Terblanche (2003).

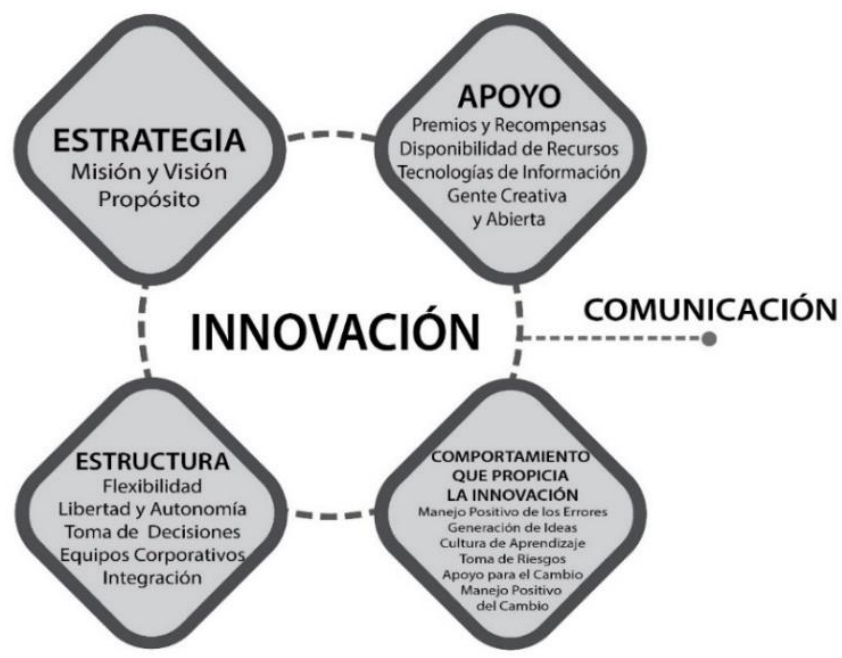

Las empresas innovadoras son las que administran el error y aprenden de ellas. Apoyando esta idea, O’Reilly (1989) describe seis normas que pueden propiciar la innovación por medio del fomento a la creatividad e implementación en las organizaciones:

- Tomar riesgos;

- Generar apertura a ideas nuevas;

- Recompensar el cambio;

- Generar objetivos comunes;

- Permitir la autonomía;

- Creer en las acciones que se llevarán a cabo. 
Gisbert (2005) menciona que el estudio e impulso de la innovación en las organizaciones implica abordar el mismo desde una perspectiva multidimensional, en las cuales es de vital importancia considerar tres variables:

- El clima creativo: buscar el compromiso de los colaboradores a través de un liderazgo participativo que fomente, apoye y valore las aportaciones de los mismos bajo un ambiente de confianza mutua.

- El compromiso estratégico con la innovación: adoptando patrones de comportamiento que traduzcan la importancia asignada a la innovación, abordando el cambio desde diferentes perspectivas, de forma permanente y asignando los recursos necesarios para lograr los objetivos.

- Soporte estructural: dotando de dinamismo y flexibilidad propios de una estructura orgánica que proporcione un sistema adecuado para el correcto desarrollo de la actividad innovadora.

Finalmente, desde una visión centrada en los miembros de la organización y en el papel fundamental de las personas en la innovación y creatividad, sus esfuerzos y compromisos con la innovación son cruciales en la construcción de organizaciones capaces de lograr que la innovación y creatividad fluyan de forma natural (Souto, 2015).

\subsection{Empowerment e Innovación}

El interés por el estudio del empowerment en el ámbito de las prácticas organizacionales aparece cuando las organizaciones comienzan a necesitar trabajadores con mayor capacidad e innovación debido al ambiente de cambio continuo y globalización en el que se encuentran inmersas en la actualidad (Jaimez \& Bretones, 2011).

De acuerdo con Cardona (2001), la confianza y el compromiso de las personas son condición necesaria para los procesos de innovación y adaptación que caracterizan a las empresas exitosas.

Jung, Chow y Wu (2003) sugieren que el empowerment está positivamente relacionado con la innovación, una vez que exista aproximación entre la alta gerencia y los colaboradores, promoviendo una cultura organizacional innovadora. Sus investigaciones, basadas en treinta y dos empresas de Taiwán, proporcionan información relevante 
afirmando que la innovación directa e indirectamente puede ser generada en la empresa mediante la creación de una cultura organizativa en la cual se motive a los empleados a discutir y probar ideas libremente.

Existe un vínculo positivo entre el empoderamiento y la innovación organizativa, esto implica que, a medida que las empresas más deleguen y den autonomía a sus empleados, son más innovadoras (Jung et al., 2003).

Pinto (2015), en una investigación de una unidad productiva del Grupo Ikea Industry en Portugal, se propuso entender el contexto del empowerment y, si está directamente relacionado con la innovación. Una de las conclusiones emergentes en los ámbitos de procesos de innovación es que, el grado de colaboración o feedback entre el nivel más operacional de una empresa y la alta dirección es uno de los factores más importantes.

Así mismo, Pinto (2015) concluyó que el empowerment puede funcionar como fuente de innovación, que su aplicación es claramente una forma de alcanzar la ventaja competitiva y que es una herramienta que, cuando es bien aplicado, esto es, adecuadamente a los objetivos y contextos organizacionales, tiene un enorme potencial para mejorar la capacidad de respuesta de las organizaciones a las nuevas realidades que se presentan diariamente.

La innovación es generadora de un cambio efectivo cuanto mayor sea el círculo de participación, esto es, cuanto más personas, grupos y organizaciones se responsabilicen de un objetivo común (Cardoso \& Pardal, 2015).

Es así que, Ford y Randolph (1992) propusieron que el empowerment es importante para mejorar la innovación. De este modo, atributos como la comunicación e información compartida hasta el nivel más operacional, la participación de los colaboradores en la toma de decisiones y una visión organizacional compartida son igualmente elementos promotores de la innovación (Ahmed, 1998).

Por su parte, Berraies, Chaher y Yahia (2014), en un estudio empírico realizado con una muestra de 248 empresas pertenecientes al sector TIC de Tunes, concluyeron que el empowerment de los colaboradores influye positivamente en la confianza, la innovación y el desempeño organizacional. En la misma investigación, se evidencia una fuerte 
relación entre el empoderamiento del empleado y la innovación organizacional.

Así mismo, Thamizhmanii y Hasan (2010), afirmaron que el empoderamiento aumenta la colaboración entre los empleados y éste impulsa la innovación.

De hecho, dando a los empleados la oportunidad de ser independientes, tener más poder y participar en la toma de decisiones, éstos se sienten más valorados y podrían estar más dispuestos a hacer un mayor esfuerzo y ser más proactivos. Pueden experimentar nuevas formas de pensar, crear nuevos conocimientos y generar ideas originales que promueven la innovación (Berraies, Chaher \& Yahia, 2014).

Por su parte, Guay, Simard y Tremblay (2000), afirmaron que una cultura de empowerment alienta a los empleados a sentirse más importantes, responsables y libres de utilizar sus inteligencias y competencias. Ésta práctica permite a las empresas explotar los conocimientos y las competencias de los empleados.

\section{METODOLOGÍA}

\subsection{Objetivos}

Conocer el nivel de empowerment estructural de los colaboradores de organizaciones con características innovadoras; identificar el grado de empowerment estructural de los colaboradores en las siguientes dimensiones: oportunidad, información, apoyo y recursos; conocer la relación existente entre las cuatro dimensiones del empowerment estructural; verificar si el nivel de empowerment estructural, en las diferentes dimensiones, varía en función de las características personales y profesionales de los colaboradores.

\subsection{Población y muestra}

Para esta investigación fue utilizado el método de muestreo no probabilístico por conveniencia, dada la conveniente proximidad y accesibilidad de los sujetos. Este método se caracteriza por ser realizada debido a la facilidad, estando los sujetos escogidos presentes en un local y momento determinado (Fortin, 1999). 
La muestra está constituida por 90 colaboradores de 3 empresas caracterizadas como innovadoras, que respondieron los ítems del cuestionario CWEQ-II de empowerment estructural. A continuación presentamos una tabla comparativa sobre los resultados obtenidos en cuanto a la evaluación de empresas en las diferentes dimensiones mencionadas anteriormente (Tabla 1). Se evaluaron un total de siete empresas y tres de ellas presentaron características innovadoras (Empresas A, B y C).

\section{Tabla I}

Resultados de evaluación de la capacidad innovadora de las empresas

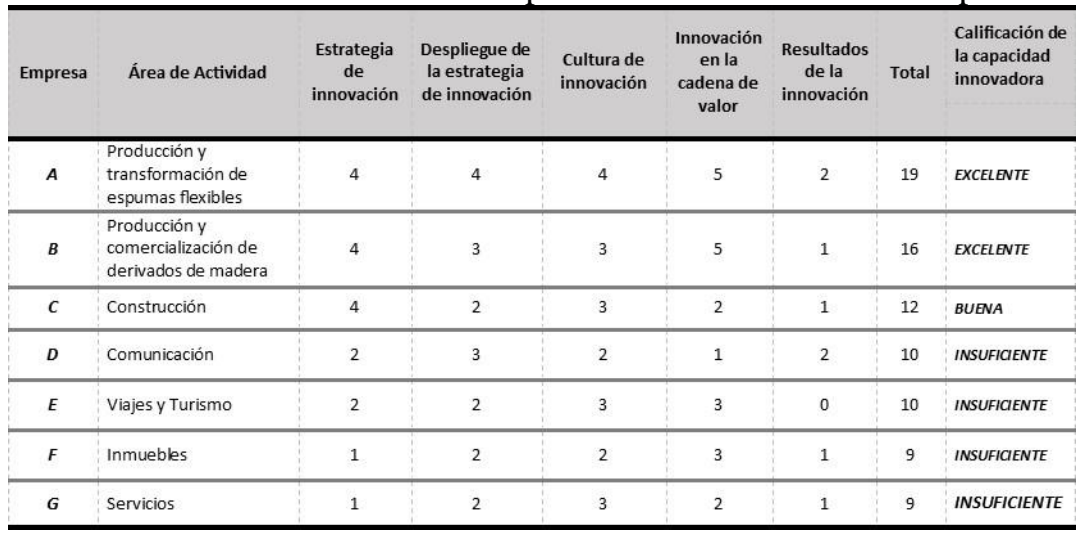

Verificamos que, en la distribución de los colaboradores en estudio en función del rango de edad (Tabla 2), un 28,9\% corresponden a edades comprendidas entre 39 y 48 años, de la misma manera, el 28,9 \% se sitúa entre edades comprendidas entre 49 y 58 años.

Seguidamente, el 23,3\% corresponde a edades comprendidas entre 19 y 28 años, el 13,3\% pertenecen al rango de 29 a 38 años y, por último, un $5,6 \%$ a los comprendidos entre 59 y 68 años de edad. 


\section{Tabla II}

Distribución de los participantes según la edad

\begin{tabular}{|l|l|l|}
\hline Edad & $\mathbf{N}$ & $\mathbf{\%}$ \\
\hline $\mathbf{1 9}-\mathbf{2 8}$ & 21 & 23.3 \\
\hline $\mathbf{2 9}-\mathbf{3 8}$ & 12 & 13.3 \\
\hline $\mathbf{3 9}-\mathbf{4 8}$ & 26 & 28.9 \\
\hline $\mathbf{4 9}-\mathbf{5 8}$ & 26 & 28.9 \\
\hline $\mathbf{5 9}-\mathbf{6 8}$ & 5 & 5.6 \\
\hline Total & $\mathbf{9 0}$ & $\mathbf{1 0 0 . 0}$ \\
\hline Mínimo & 19 & \\
\hline Máximo & 63 & \\
\hline Media & 41.24 & \\
\hline Moda & 54 & \\
\hline Desv.Estándar & 12.16 & \\
\hline
\end{tabular}

\subsection{Instrumentos}

Conforme al modelo planteado para esta investigación, el mismo está constituido dos cuestionarios distintos para lograr los objetivos propuestos.

El primero es el Test de Innovación Empresarial ICT desarrollado por el Instituto Catalán de Tecnología (1999), que consiste en un test de evaluación rápida e integral para determinar en qué medida una empresa es innovadora o no. El mismo es uno de los más utilizados y teniendo en cuenta las experiencias con buenos resultados en otras investigaciones ya realizadas, consideramos que, este test se adapta perfectamente a los objetivos de nuestro estudio ya que el mismo está disponible para su utilización para empresas y/o investigaciones.

Una vez que hemos seleccionado este instrumento, procedimos a su adaptación y traducción a la lengua portuguesa con ayuda de profesionales expertos en lengua portuguesa e intercomprensión lingüística.

Este test permite evaluar el nivel de innovación de las empresas teniendo en cuenta 5 aspectos fundamentales:

- Estrategia de innovación;

- Despliegue de la estrategia de innovación;

- Cultura de la innovación; 
- Innovación en la cadena de valor;

- Resultados de la innovación.

Como nuestra investigación pretende conocer el nivel de empowerment estructural que se presentan en empresas con características innovadoras, decidimos a utilizar un segundo cuestionario para determinar el nivel de empowerment estructural en cuatro dimensiones:

- Oportunidad;

- Información;

- Apoyo;

- Recursos.

Una vez evaluado el nivel de innovación de las empresas, se aplicaron a colaboradores de aquellas que reunían las características de ser innovadoras (Empresas A, B y C) el segundo instrumento para medir el nivel de empowerment estructural, dividido en dos partes; la primera referida a la caracterización sociodemográfica y profesional de los colaboradores $\mathrm{y}$, la segunda, respecto al nivel de empowerment estructural.

Este cuestionario fue presentado a los colaboradores con una escala tipo Likert de uno al cinco, en orden creciente, donde el menor valor es poco (uno) y el mayor valor es mucho (cinco).

Tal instrumento es el Cuestionario para Condiciones de Efectividad en el Trabajo CWEQ-II (Conditions of Work Effectiveness Questionnaire), desarrollado por Laschinger et al. (2004), traducido y adaptado a la población portuguesa por Orgambídez-Ramos et al. (2015). 


\section{Tabla III}

Versión en inglés y portugués del CWEQ-II

\begin{tabular}{|c|c|}
\hline Inglés & Portugués \\
\hline $\begin{array}{l}\text { Opportunity } \\
\text { How much of each kind of } \\
\text { opportunity do you have in your } \\
\text { present job? } \\
\text { 1. Challenging work } \\
\text { 2. The chance to gain new skills } \\
\text { and knowledge on the job } \\
\text { 3. Tasks that use all of your own } \\
\text { skills and knowledge } \\
\text { Information } \\
\text { How much access to information } \\
\text { do you have in your present job? } \\
\text { 4. The current state of the } \\
\text { company } \\
\text { 5. The values of top management } \\
\text { 6. The goals of top management } \\
\text { Support } \\
\text { How much access to support do } \\
\text { you have in your present job? } \\
\text { 7. Specific information about } \\
\text { things you do well } \\
\text { 8. Specific comments about } \\
\text { things you could improve } \\
\text { 9. Helpful hints or problem } \\
\text { solving advice } \\
\text { Resources } \\
\text { How much access to resources do } \\
\text { you have in your present job? } \\
\text { 10. Time available to do } \\
\text { necessary paperwork } \\
\text { 11. Time available to accomplish } \\
\text { job requirements } \\
\text { 12. Acquiring temporary help } \\
\text { when needed }\end{array}$ & $\begin{array}{l}\text { Oportunidade } \\
\text { Em que medida tem cada uma das } \\
\text { oportunidades? } \\
\text { 1. Trabalho desafiante } \\
\text { 2. A possibilidade para } \\
\text { desenvolver novas competências } \\
\text { e conhecimentos } \\
\text { 3. Tarefas que usam todas as } \\
\text { suas próprias competências e } \\
\text { conhecimentos } \\
\text { Informação } \\
\text { Até que ponto tem acesso à } \\
\text { informação relativa a? } \\
\text { 4. Estado atual da empresa } \\
\text { 5. Os valores da gestão de topo } \\
\text { 6. Os objetivos da gestão de topo } \\
\text { Suporte } \\
\text { Até que ponto tem acesso às? } \\
\text { 7. Informações específicas sobre } \\
\text { coisas que faz bem } \\
\text { 8. Comentários específicos sobre } \\
\text { aspetos que poderia melhorar } \\
\text { 9. Dicas úteis e conselhos de } \\
\text { resolução de problemas } \\
\text { Recursos } \\
\text { Em termos de recursos, em que } \\
\text { medida tem? } \\
\text { 10. Tempo disponível para tratar / } \\
\text { organizar os papéis } \\
\text { 11. Tempo disponível para } \\
\text { cumprir exigências de trabalho } \\
\text { 12. Ajuda temporária quando } \\
\text { necessária }\end{array}$ \\
\hline
\end{tabular}


El análisis de consistencia de la escala de empowerment estructural fue estimado a partir del coeficiente Alpha de Cronbach mediante el uso del programa SPSS versión 22.0. El cálculo de éste coeficiente nos permite determinar el grado de efectividad de cada ítem para medir cada dimensión.

Para las cuatro dimensiones testeadas, los valores se encontraron por encima del nivel considerado aceptable (0.70), específicamente, oportunidad (0.86), información (0.88), apoyo $(0.88)$ y recursos $(0.89)$, por lo que se puede concluir que en la presente muestra, la escala presenta buena consistencia ya que cada ítem que lo compone, se presenta como un todo homogéneo.

Como podemos verificar en la tabla 4, los valores que hemos obtenido, son ligeramente superiores, en todas las dimensiones, a los obtenidos por el autor principal.

\section{Tabla IV}

Análisis de fidelidad de las dimensiones del CWEQ-II utilizado, comparativamente con la escala original validada por Laschinger et al.

(2004)

\begin{tabular}{|c|c|c|c|}
\hline \multicolumn{2}{|l|}{$\begin{array}{l}\text { Escala } \\
\text { utilizada por } \\
\text { Laschinger } \\
\text { et al. (2004) }\end{array}$} & \multicolumn{2}{|c|}{$\begin{array}{l}\text { Escala utilizada en } \\
\text { la investigación }\end{array}$} \\
\hline Scale & Alpha Cronbach & Dimensión & Alpha Cronbach \\
\hline Opportunity & 0.79 & Oportunidad & 0.86 \\
\hline Information & 0.87 & Información & 0.88 \\
\hline Support & 0.79 & Aроуо & 0.88 \\
\hline Resources & 0.79 & Recursos & 0.89 \\
\hline
\end{tabular}

Una vez realizada la investigación bibliográfica y durante el desarrollo del marco teórico de esta investigación, primeramente se procedió a solicitar la autorización al autor correspondiente para la utilización del CWEQ-II, traducido y adaptado a la lengua portuguesa por OrgambídezRamos et al. (2015). Después de preparar y testear los instrumentos a ser utilizados, se procedió a la construcción de una plataforma on line de recolección de datos de manera a que la aplicación sea más flexible y práctica. 
Seguidamente, fueron enviados y solicitados vía email, a directores y jefes de determinadas áreas de empresas del distrito de Viseu, Portugal, la autorización para poder aplicar el Test de Innovación empresarial ICT. Fueron enviadas en total, solicitudes a 100 empresas, de las cuales respondieron solo 7. Las solicitudes fueron hechas a través de una exposición formal en donde se refirió a la temática del estudio, punto de partida, objetivos. En la misma aseguramos el tratamiento con discreción de los datos proveídos por las empresas, ya que nuestra investigación tiene un carácter exclusivamente académico.

Una vez recibidos los tests y analizados, procedimos a evaluar la capacidad innovadora de estas empresas, así, seleccionamos 3 empresas innovadoras y aplicamos el siguiente cuestionario a sus colaboradores.

Y finalmente, vía email enviamos el cuestionario anexado de empowerment estructural y el link para que pueda ser completado on line, en el caso que las empresas consideraran pertinente, así como también todas las instrucciones correspondientes para el llenado de los mismos.

\section{ANÁLISIS Y RESULTADOS}

De manera a percibir mejor, en cuales dimensiones de la escala los colaboradores presentan mayores niveles de empowerment estructural y al mismo tiempo, ir al encuentro de nuestro objetivo de estudio, identificar el grado de empowerment estructural de los colaboradores de organizaciones con características innovadoras en las siguientes dimensiones: oportunidad, información, apoyo y recursos, elaboramos la tabla 5 en donde cada ítem fue agrupado en su dimensión correspondiente. A partir de cada ítem correspondiente, fueron agrupadas junto con otras que respondían a una dimensión común, conforme a la escala original. Así surgieron cuatro nuevas variables para su mejor estudio y comprensión a través del software SPSS versión 22.0.

Tales variables corresponden a las dimensiones que nos permitirán evaluar el grado de empowerment estructural (oportunidad, información, apoyo y recursos).

Así, analizando la tabla podemos observar que la dimensión "recursos" presenta la menor media (2.87), lo que significa que los 
colaboradores presentan un nivel de empowerment por debajo de la media.

En cuanto a las dimensiones "oportunidad" con una media de 3.42, "información" con una media de 3.09 y "apoyo" con una media de 3.26, estas presentan valores levemente superior a la media y por lo tanto, el nivel de empowerment de los colaboradores es levemente superior en estas tres dimensiones.

\section{Tabla V}

Estadística descriptiva de las dimensiones del empowerment estructural

Estadística Descriptiva

N Mínimo
Máximo

Media
Desviación

Estándar

\begin{tabular}{llllll} 
Oportunidad & 90 & 1.33 & 5.00 & 3.42 & .860 \\
\hline Información & 90 & 1.00 & 5.00 & 3.09 & .938 \\
\hline Apoyo & 90 & 1.00 & 5.00 & 3.26 & .824 \\
\hline Recursos & 90 & 1.00 & 5.00 & 2.87 & .972 \\
\hline
\end{tabular}

(listwise)

Una vez finalizado el análisis descriptivo de los datos correspondientes, procedimos a verificar otros objetivos de investigación formulados mediante el análisis inferencial de los datos.

Previamente, procedimos a verificar el presupuesto de normalidad de distribución de valores de las variables en estudio, en varias categorías respecto a las características personales y profesionales.

La verificación se realizó a través del test de Kolmogorov-Smirnov. Este contraste se realiza para comprobar si se verifica la hipótesis de normalidad necesaria para que el resultado de algunos análisis sea fiable. Como se verificó la presunción de normalidad en casi la totalidad de los datos, entonces procedimos a la aplicación tests paramétricos.

El objetivo: conocer la relación existente entre las cuatro dimensiones del empowerment estructural, fue analizado a través de la utilización de la técnica más apropiada para nuestra muestra (Coeficiente de Pearson), teniendo en cuenta las cuatro variables o dimensiones clave de nuestra investigación: oportunidad, información, apoyo y recursos. De la lectura y análisis de los datos de la tabla 6 , verificamos que existen correlaciones 
estadísticamente significativas y positivas entre todas las dimensiones estudiadas.

De este modo, haciendo una comparación entre las dimensiones apoyo y recursos $[\mathrm{r}=0.722(\mathrm{p}=0.000<0.01)]$, estos presentan el mayor nivel de relación y podemos concluir que, a medida que las empresas u organizaciones proveen mayor acceso a recursos a sus colaboradores, ellos percibirán un mayor apoyo o soporte.

Por otra parte, teniendo en cuenta las dimensiones oportunidad y apoyo $[\mathrm{r}=0.701(\mathrm{p}=0.000<0.01)]$, estos presentan el segundo mayor nivel de relación, por lo que podemos concluir que, a medida que los colaboradores reciben mayor feedback sobre su desempeño en la empresa, ellos sentirán una mayor oportunidad para desarrollarse y aplicar todos sus conocimientos en la empresa.

Finalmente, si observamos las dimensiones información y apoyo $[\mathrm{r}=0.632(\mathrm{p}=0.000<0.01)]$, también presentan una relación positiva y podemos afirmar que, cuanto más informaciones referente a la empresa reciban los colaboradores, ellos sentirán mayor apoyo y respaldado respectivamente. 


\section{Tabla VI}

Correlación de Pearson para las dimensiones oportunidad, información, apoyo y recursos

\begin{tabular}{|c|c|c|c|c|c|}
\hline \multicolumn{6}{|l|}{ Correlaciones } \\
\hline & & Oportunidad & Información & Apoyo & Recursos \\
\hline \multirow{3}{*}{ Oportunidad } & $\begin{array}{l}\text { Pearson Cor- } \\
\text { relation }\end{array}$ & 1 & $.538^{* *}$ & $.701^{* *}$ & $.581^{* *}$ \\
\hline & Sig. (2-tailed) & & .000 & .000 & .000 \\
\hline & $\mathrm{N}$ & 90 & 90 & 90 & 90 \\
\hline \multirow{3}{*}{ Información } & $\begin{array}{l}\text { Pearson Cor- } \\
\text { relation }\end{array}$ & $.538^{* *}$ & 1 & $.632^{* *}$ & $.578^{* *}$ \\
\hline & Sig. (2-tailed) & .000 & & .000 & .000 \\
\hline & $\mathrm{N}$ & 90 & 90 & 90 & 90 \\
\hline \multirow{3}{*}{ Apoyo } & $\begin{array}{l}\text { Pearson Cor- } \\
\text { relation }\end{array}$ & $.701^{* *}$ & $.632^{* *}$ & 1 & $.722^{* *}$ \\
\hline & Sig. (2-tailed) & .000 & .000 & & .000 \\
\hline & $\mathrm{N}$ & 90 & 90 & 90 & 90 \\
\hline \multirow{3}{*}{ Recursos } & $\begin{array}{l}\text { Pearson Cor- } \\
\text { relation }\end{array}$ & $.581^{* *}$ & $.578^{* *}$ & $.722^{* *}$ & 1 \\
\hline & Sig. (2-tailed) & .000 & .000 & .000 & \\
\hline & $\mathrm{N}$ & 90 & 90 & 90 & 90 \\
\hline
\end{tabular}

**Correlación es significativa al nivel de 0.01

Al analizar nuestro último objetivo: verificar si el nivel de empowerment estructural, en las diferentes dimensiones, varía en función de las características personales y profesionales de los colaboradores, recurrimos a la utilización de test paramétricos, en este caso ANOVA y el T-test según los tipos de variables involucradas.

En este sentido, determinamos la existencia de diferencias significativas con respecto a la experiencia profesional de los colaboradores y el tiempo de trabajo en la empresa, en las demás características personales y profesionales no se presentaron relación alguna.

Al dar una lectura y análisis a la tabla 7 , verificamos que existen diferencias estadísticas significativas del empowerment estructural en la 
dimensión oportunidad $(\mathrm{p}=0.012<0.05)$ con respecto a los colaboradores que tienen experiencia profesional de 0 a 9 años $(M=3.75)$.

Así, teniendo en cuenta estos resultados, podemos concluir que los colaboradores que tienen menor experiencia profesional son aquellos que presentan un mayor nivel de empowerment estructural.

\section{Tabla VII}

Test ANOVA del empowerment estructural respecto a la experiencia profesional

\begin{tabular}{lcllll}
\hline \multicolumn{4}{c}{ ANOVA } & & \\
\hline Tiempo Exp. & $N$ & Media & $\begin{array}{l}\text { Desviación } \\
\text { Estándar }\end{array}$ & $F$ & $p$
\end{tabular}

\section{Profesional}

(Años)

\begin{tabular}{|c|c|c|c|c|c|c|}
\hline \multirow[t]{4}{*}{ Oportunidad } & $0-9$ & 20 & 3.75 & .836 & \multirow[t]{4}{*}{3.903} & \multirow[t]{4}{*}{$.012 *$} \\
\hline & $10-19$ & 22 & 3.74 & .658 & & \\
\hline & $20-29$ & 30 & 3.14 & .704 & & \\
\hline & $30-49$ & 18 & 3.16 & 1.115 & & \\
\hline \multirow[t]{4}{*}{ Información } & $0-9$ & 20 & 3.31 & .894 & \multirow[t]{4}{*}{.549} & \multirow[t]{4}{*}{.650 } \\
\hline & $10-19$ & 22 & 3.07 & .991 & & \\
\hline & $20-29$ & 30 & 3.04 & .916 & & \\
\hline & $30-49$ & 18 & 2.94 & .991 & & \\
\hline \multirow[t]{4}{*}{ Apoyo } & $0-9$ & 20 & 3.55 & .811 & \multirow[t]{4}{*}{1.513} & \multirow[t]{4}{*}{.217} \\
\hline & $10-19$ & 22 & 3.31 & .793 & & \\
\hline & $20-29$ & 30 & 3.05 & .783 & & \\
\hline & $30-49$ & 18 & 3.22 & .907 & & \\
\hline \multirow[t]{4}{*}{ Recursos } & $0-9$ & 20 & 3.25 & .978 & \multirow[t]{4}{*}{1.775} & \multirow[t]{3}{*}{.158} \\
\hline & $10-19$ & 22 & 2.86 & 979 & & \\
\hline & $20-29$ & 30 & 2.61 & .849 & & \\
\hline & $30-49$ & 18 & 2.88 & 1.084 & & \\
\hline
\end{tabular}

Al observar y analizar la tabla 8 , verificamos que existen diferencias significativas respecto al empowerment estructural y el tiempo de trabajo en la empresa en la dimensión oportunidad $(\mathrm{p}=0.002<0.01)$, específicamente para aquellos sujetos que tienen un tiempo de trabajo de 0 a 9 años $(\mathrm{M}=3.78)$. 
Entonces, estos datos nos llevan a concluir que, el empowerment estructural, en la dimensión oportunidad, es mayor para aquellos sujetos que tienen menos tiempo trabajando en la empresa.

Tabla VIII

Test ANOVA del empowerment estructural respecto tiempo de trabajo en la empresa

\begin{tabular}{|c|c|c|c|c|c|c|}
\hline \multicolumn{7}{|l|}{$\overline{A N O V A}$} \\
\hline \multirow{2}{*}{\multicolumn{2}{|c|}{$\begin{array}{l}\text { Tiempo de } \\
\text { Trabajo (Años) }\end{array}$}} & \multirow{3}{*}{$\begin{array}{l}N \\
\\
35\end{array}$} & \multirow{3}{*}{$\begin{array}{r}\text { Media } \\
3.7810\end{array}$} & \multirow{3}{*}{$\begin{array}{l}\begin{array}{l}\text { Desviación } \\
\text { Estándar }\end{array} \\
.74511\end{array}$} & \multirow{3}{*}{\begin{tabular}{|l|}
$\boldsymbol{F}$ \\
6.489
\end{tabular}} & \multirow{3}{*}{\begin{tabular}{|l|}
$p$ \\
$p$ \\
$.002^{* *}$ \\
\end{tabular}} \\
\hline & & & & & & \\
\hline \multirow{3}{*}{ Oportunidad } & $0-9$ & & & & & \\
\hline & $10-19$ & 17 & 3.4510 & .66605 & & \\
\hline & $20-39$ & 38 & 3.0965 & .91994 & & \\
\hline \multirow{3}{*}{ Información } & $0-9$ & 35 & 3.2476 & 1.00112 & .994 & .374 \\
\hline & $10-19$ & 17 & 3.1176 & .96423 & & \\
\hline & $20-39$ & 38 & 2.9386 & .86596 & & \\
\hline \multirow{3}{*}{ Apoyo } & $0-9$ & 35 & 3.4857 & .78940 & 2.397 & .097 \\
\hline & $10-19$ & 17 & 3.2353 & .64296 & & \\
\hline & $20-39$ & 38 & 3.0702 & .89429 & & \\
\hline \multirow{3}{*}{ Recursos } & $0-9$ & 35 & 3.1429 & .99109 & 2.383 & .098 \\
\hline & $10-19$ & 17 & 2.6275 & .75353 & & \\
\hline & $20-39$ & 38 & 2.7281 & 1.00406 & & \\
\hline
\end{tabular}

\section{DISCUSIÓN Y CONCLUSIONES}

Hoy en día, el colaborador debe estar facultado para tomar decisiones y resolver problemas. Esto se logra a través del empowerment o empoderamiento. Empoderar ofrece el potencial de utilizar la capacidad humana para que las compañías sobrevivan en el mundo cada vez más complejo y dinámico de hoy (Blanchard et al., 1996).

Afzal, Hussain \& Gilani (2019), afirmaron que el empoderamiento en el lugar de trabajo y el liderazgo resonante mejoran el desempeño y la satisfacción laboral. Así las organizaciones que dan a sus colaboradores acceso a información, oportunidades de aprendizaje, apoyo y recursos, 
empoderan a sus colaboradores y posibilitan mayor eficiencia en el trabajo.

En lo que concierne a los resultados, podemos concluir que la muestra presentó un nivel de empowerment superior en las dimensiones evaluadas y al comparar con la escala original, podemos afirmar que, por el hecho de que provengan de empresas innovadoras, éstos presentaron una mayor percepción al nivel de empowerment estructural.

También verificamos que las cuatro dimensiones presentaron una relación significativa y estos resultados sugieren que, para que los colaboradores se sientan empoderados, las organizaciones deben darles acceso a oportunidades para aprender y desarrollarse, proveerles información y recursos necesarios y el apoyo indispensable para crecer y aportar lo mejor de sí mismos a favor de la empresa y su entorno.

\section{FUENTES Y BIBLIOGRAFÍA}

Ahmed, P. (1998). Culture and Climate for Innovation. European Journal of Innovation Management, 1(1), 30-43. https://doi.org/10.1108/14601069810199131

Arborniés, L. (2009). La disciplina de la innovación: rutinas creativas. Argentina: Díaz de Santos.

Afzal, M., Hussain, M., \& Gilani, S. A. (2019). The influence of structural empowerment and resonant leadership on job satisfaction of registered nurses at public hospital of Lahore Pakistan. International Journal of Advancements in Research \& Technology, 8(1), 15-26.

Berraies, S., Chaher, M., \& Yahia, K. B. (2014). Employee empowerment and its importance for trust, innovation and organizational performance. Business Management and Strategy, 5(2), 82-103. DOI: https://doi.org/10.5296/bms.v5i2.6558

Blanchard, K., Carlos, J., \& Randolph, A. (1996). Empowerment: tres claves para lograr que el proceso de facultar a los empleados funcione en su empresa. Colombia: Norma.

Cardoso, F., \& Pardal, I. (2015). Colaborar para Inovar: A inovação organizacional e social como resultado do processo de decisão. Lisboa: Sílabo.

Cardoso Teixeira, A., \& Barbieri-Figueiredo, M. (2015). Empoderamento 
e satisfação profissional em Enfermagem: uma revisão integrativa, em consonância com a Teoria Estrutural. Revista de Enfermagem Referência, IV (6), 151-160.

Cardona, S. (2001). Intrategia: una dimensión básica de la cultura empresarial. Madrid: McGraw Hill.

Chiavenato, I. (2009). Gestión del Talento Humano. (3 ${ }^{\text {a }}$ Edición). México: McGraw Hill.

Damanpour, F., \& Gopalakrishnan, S. (2001). The dynamics of the adoption of products and process innovations in organizations. Journal of Management Studies, 38(1), 45-65. https://doi.org/10.1111/14676486.00227

De Oslo, M. (2006). Directrices para la recogida e interpretación de información relativa a innovación. México: OCDE-Eurostat.

Dessler, G., \& Varela, R. (2011). Administración de Recursos Humanos: Enfoque Latinoamericano. (5 $5^{\mathrm{a}}$. Edición). México: Pearson Prentice Hall.

Fortin, M. (1999). O Processo de Investigação: Da Conceção à Realização. Loures: Lusociência.

Gisbert, M., \& Velasco, A. (2007). La persona protagonista de la innovación. Madrid: Gráficas Arias Montano.

Gisbert, M. (2005). Creatividad e innovación en la práctica empresarial. Madrid: Gráficas Arias Montano.

Gouveia, A. (2013). Efeitos do empowerment estrutural nos comportamentos de movilização de profissionais de saúde e na percepção dos eventos adversos associados aos cuidados aos doentes: uma revisão sistemática da literatura (Tese de Mestrado em Psicología das organizações e do trabalho). Universidade de Coimbra, Portugal.

Guay, P., Simard, G., \& Tremblay, M. (2000). Organizational and individual determinants of atypical employment: The case of multiple jobholding and self-employment (No. 2000s-26). CIRANO.

Jaimez, M. J., \& Bretones, F. D. (2011). El empowerment organizacional: el inicio de una gestión saludable en el trabajo. Revista de Trabajo y Seguridad Social. Recursos Humanos, 344, 209-232.

Jung, D. I., Chow, C., \& Wu, A. (2003). The role of transformational leadership in enhancing organizational innovation: Hypotheses and 
some preliminary findings. The Leadership Quarterly, 14(4), 525-544. https://doi.org/10.1016/S1048-9843(03)00050-X

Laschinger, H. K. S., Finegan, J. E., Shamian, J., \& Wilk, P. (2004). A longitudinal analysis of the impact of workplace empowerment on work satisfaction. Journal of Organizational Behavior, 25(4), 527545. https://doi.org/10.1002/job.256

Orgambídez-Ramos, A., Gonçalves, G., Santos, J., Borrego-Alés, Y., \& Mendoza-Sierra, M. I. (2015). Empowering Employees: A Portuguese Adaptation of the Conditions of Work Effectiveness Questionnaire (CWEQ-II). Revista Psicología, 29(1), 1-10.

Orgambídez-Ramos, A., Borrego-Alés, Y., \& Ruiz-Frutos, C. (2018). Empowerment, vulnerabilidad al estrés y burnout en enfermeros portugueses. Ciência \& Saúde Coletiva, 23, 259-266.

Miles, R., \& Snow, C. (1978). Organizational strategy, structure and process. New York: McGraw Hill.

O' Reilly, C. (1989). Corporations, Culture and Commitment: motivation and social control in organizations. California Management Review, 31(4), 9-25. Pfeiffer, I., \&

Pinto, B. (2015). Empowerment e Inovação: Estudo de Caso Ikea Industry, Paços de Ferreira (Tese de Mestrado em Economia e Gestão da Inovação). Universidade do Porto, Portugal.

Ribeiro, C., \& Almada, F. (2001). Empowerment: ciclo de implementação, dimensões e tipologia. Gestão e Produção, 8(3), 237249.

Rogers, E. (1983). Diffusion and Innovation. New York: Free Press.

Slack, N., Chambers, S., Harland, C., Harrison, A., \& Johnston, R. (1997). Administração da Produção. São Paulo: Atlas.

Souto, J. (2015). Gestión de una Cultura de Innovación Basada en las Personas. Journal of Technology Management \& Innovation, 10(3), 60-65.

DOI: $10.4067 / \mathrm{S} 0718-27242015000300007$

Spreitzer, G. (2008). Taking Stock: A Review of More Than Twenty Years of Research on Empowerment at Work. In J. Barling, \& C. Cooper, The SAGE Handbook of Organizational Behavior (pp. 54-72). Londres: SAGE Publications.

Thamizhmanii, S., \& Hasan, S. (2010). A review on an employee 
empowerment in TQM practice. Journal of Achievements in Materials and Manufacturing Engineering, 39(2), 204-210.

Wilson, T. (1996). Manual del Empowerment: Cómo conseguir lo mejor de sus colaboradores. Barcelona: Gestión 2000.

Wilkinson, A. (1998). Empowerment: theory and practice. Personnel Review, 27(1), 40-56 